Resnick is distinguished above all by the clarity of expression and the very full verbal arguments given for each stage in the theory. It is written with the reader in mind who is not going to be taught the subject, but who must learn it for himself as he goes along. The treatment is fairly orthodox, and care is taken not to go too far in such a small space. It begins with the Michelson-Morley experiment and proceeds in the obvious way to the Lorentz transformation. The application to optics (aberration and the Doppler effect) leads on to dynamics, where the new concept of momentum is justified by considering collisions. Following this there is a chapter on electromagnetism and a brief discussion on possible limitations of special relativity on the very small scale. Each chapter is completed with a set of questions. As well as the main chapters there are three supplementary topics at the back, one on space-time diagrams, one on the clock paradox (which is very clear, but it is no doubt too much to expect that it will have disposed of the arguments about this), and one of four pages showing the way forward to general relativity. There is an excellent index.

Dr Shadowitz, on the other hand, is putting forward a very distinctive point of view. His actual treatment of the theory follows surprisingly similar lines to that of Resnick, beginning with the Michelson-Morley experiment and going on to consider space and time contractions, the optical effects, mechanics and electromag. netism in turn. Rather oddly, his book concludes with a chapter on experimental evidence, after having discussed the elements of four-dimensional tensor analysis. His different approach, however, is in the reliance which he places on certain space-time diagrams. In particular, he considers in great detail the Loedel diagram, which is essentially that got by using inclined axes to represent contravariant vectors. Here space and time are real co-ordinates, just as they are in the diagram which is usually employed, but the effect of Lorentz transformations is made explicit more easily. This is contrasted with the so-called Brehme diagrams which is the corresponding one for covariant vectors. Neither of these diagrams is the same as the original (real) Minkowski diagram, because in the Minkowski diagram one observer is treated as preferred over all the others, having his axes at rightangles, whereas in the Loedel and the Brehme diagrams the observers are treated symmetrically. It is a subjective matter whether someone learning the subject finds it easier to do it from a geometrical or from an algebraic point of view, but certainly the diagrams used in this book have considerable advantage over the Minkowski diagram, which it is eustomary to use. In an appendix the author also gives nomographs for the composition of velocities parallel or at right-angles, and for transformation of momenta and kinetic energies. These are extremely useful in working out practical examples. Other appendices deal with somewhat more technical questions in electromagnetism. The index is somewhat fuller than Resnick's and the whole book is produced with considerable elegarce.

C. W. Kilmister

\section{ELEMENTAL DATA}

\section{Handbook of the Physicochemical Properties of the} Elements

Edited by G. V. Samsonov. Translated from the Russian. Pp. xii +941 . (New York: IFI/Plenum Data Corporation, 1968.) $\$ 40$.

THe Russian edition of this book, now three years old, has been added to in translation to include a number of new tables, and it deals quite specifically with elements; no compounds are referred to.

About one quarter of the book contains information pertaining to atomic and nuclear structure and properties.
This includes spectroscopic and electronic as well as the more usual structural data. The remainder of the first half consists of tables of thermodynamic, heat, electrical, magnetic, optical and mechanical properties.

The second half deals with electrochemical properties such as electrode potentials and oxidation reduction potentials, and there is a very large chapter on chemical properties as far as reactions with a large selection of reagents, some common, some very uncommon.

The whole volume contains more than 2,500 references and is a quite remarkable assembly of factual information. It is an expensive book, but not unduly so when its size and detailed scope are considered. There will be few individuals who will be able to justify this as an addition to their bookshelves, but many laboratories will find this ready source of elemental data a valuable library addition. J. C. Rовв

\section{THEORETICIANS AND DESIGNERS}

\section{Optimization in Control Theory and Practice}

By I. Gumowski and C. Mira. Pp. viii + 242. (London: Cambridge University Press, 1968.) 65s.; $\$ 10.50$.

IT appears that there are now two cultures growing apart within the relatively narrow field of control. These might be called theory and practice, and the people concerned are the theoreticians (applied mathematicians) and the designers (physicists, chemists and engineers). For rapid advancement of the subject much communication is required between the two classes of specialist. The theoreticians should give simple and full accounts of their theories rather than accounts which are meant to impress and confound. In return the designers should continually indicate the areas in which theoretical advance is required.

The two authors of this book are obviously theoreticians and they state that a prime aim in writing it was to stimulate the exchange of ideas between the two classes. In this respect, theoreticians who read the early pages may be moved to ask themselves whether their own researches have any practical significance. Here the authors warn against non-essential generalizations and "optimum" systems which may be too sensitive to plant variations or to the theoretical approximations which have been made. However, it is doubtful whether the main body of the book will do anything to encourage the dialogue between the two classes.

Most of the book consists of what is undoubtedly a very learned discussion of variational methods of optimization. The approach is classical and it is based on the work of mathematicians such as Euler, Lagrange and Hamilton. Particular prominence is given to the methods of Carathéodory. In so doing, both Bellman and Pontryagin are reflected in poor light. This is best illustrated by the following quotations from the book: "Except for the terminology, all features of dynamic programming can already be found in the work of Massé", "Dynamic programming is therefore a particular case of the Carathéodory formulation, and the particularity of dynamic programming consists only in the rather involved and circuitous method of deriving equation (4.18)", and "Both dynamic programming and the maximum principle are less general than the theory of the Carathéodory formulation", where here "the maximum principle" is due to Pontryagin.

The final chapter of the book is devoted to approximate methods of computing extremal solutions. In this and earlier chapters there is a distinct lack of examples and none which appears to have immediate practical application. Nevertheless, the book will be useful for reference for anyone making a deep study of variational methods of optimization. There is an extensive bibliography to which frequent and specific reference is made throughout 